\title{
Exact Green's Function for the Finite Rectangular Potential Well in One Dimension
}

\author{
R. Baltin \\ Department of Theoretical Chemistry, University of Ulm, Ulm, FRG
}

Z. Naturforsch. 40 a, 379-382 (1985); received August 24, 1984

For the one-dimensional potential well with finite height $V_{0}\left(V_{0}>0\right.$ or $\left.V_{0}<0\right)$ the exact Green's function $G$ is calculated by solving the differential equation. The poles of $G$ in the complex energy plane are shown to coincide with the solutions to the Schrödinger eigenvalue equation for this potential. The well-known Green's functions for the special cases of the free particle and of the particle in an infinitely high potential box are recovered.

Green's functions are of great importance in quantum physics for calculating energy eigenvalues, eigenfunctions, density of states [1] or, via inverse Laplace transformation, for the canonical density matrix in statistical mechanics [2]. Furthermore, the Green's function is a valuable tool for perturbation theory, e.g. in scattering problems [3].

For use as model system or for test of approximation methods it is always advantageous to have exact solutions to certain quantum mechanical potentials available. Exact solutions are known for free particles under various boundary conditions including the particle in a box with infinitely high walls, see e.g. $[4,5]$. Further exactly solvable potentials are the harmonic oscillator in one, three or more dimensions $[6-9,15]$, the Morse-, Kratzerand double minimum potential [7], the non-relativistic Coulomb potential $[10-12]$, and the homogeneous magnetic field [13,14]. Surprisingly, however, the case of a particle moving in a one-dimensional rectangular potential well (or barrier) with finite heigh $V_{0}$ seems not to have been dealt with up to now although this potential is

- still relatively simple and therefore is tractable from the mathematical point of view

- non-trivial from a physical point of view inasmuch as both bound and unbound states can occur if $V_{0}<0$.

There are mainly three methods for calculating the Green's function $G\left(x, x_{0}, z\right)[1,4,5]$.

Reprint requests to Prof. Dr. R. Baltin, Abteilung für Theoretische Chemie der Universität, Oberer Eselsberg, D-7900 Ulm.

\section{Solving the Differential Equation}

$$
\left(z-\hat{H}_{x}\right) G\left(x, x_{0}, z\right)=\delta\left(x-x_{0}\right)
$$

where $z$ is a complex parameter,

$$
\hat{H}_{x} \equiv-\frac{\hbar^{2}}{2 m} \frac{\partial^{2}}{\partial x^{2}}+V(x)
$$

and $G$ has to satisfy certain boundary conditions.

\section{Summing up the Spectral Representation}

$$
G\left(x, x_{0}, z\right)=\sum_{n=1}^{\infty} \frac{\psi_{n}^{*}\left(x_{0}\right) \psi_{n}(x)}{z-E_{n}},
$$

where $E_{n}$ and $\psi_{n}$ are eigenvalues and eigenfunctions of $\hat{H}_{x}$, respectively. If the spectrum of $\hat{H}_{x}$ has continuous parts, the summation has to be replaced by an integration over these parts.

\section{Performing the Feynman Path Integral Expression for the Green's Function, see [16, 17]}

In this paper the first approach is adopted for the potential

$$
V(x)=\left\{\begin{array}{l}
V_{0}(>\text { or }<0) \quad \text { if } \quad 0 \leqq x \leqq L, \\
0 \text { else }
\end{array}\right.
$$

According to the general method [4,5], two solutions of the homogeneous equation

$$
\left(z-\hat{H}_{x}\right) G_{h}=0
$$

have to be determined, $G_{h}^{>}(x, z)$ and $G_{h}^{<}(x, z)$, such that

$$
\begin{aligned}
& G_{h}^{>}(x, z) \text { bounded for } x \rightarrow+\infty, \\
& G_{h}^{<}(x, z) \text { bounded for } x \rightarrow-\infty .
\end{aligned}
$$

0340-4811/ 85 / 0400-0379 \$ 01.30/0. - Please order a reprint rather than making your own copy. 
Then the desired Green's function is given by

$$
\begin{aligned}
G\left(x, x_{0}, z\right)= & \frac{2 m}{\hbar^{2} W(z)}\left[\theta\left(x_{0}-x\right) G_{h}^{<}(x, z) G_{h}^{>}\left(x_{0}, z\right)\right. \\
& \left.+\theta\left(x-x_{0}\right) G_{h}^{>}(x, z) G_{h}^{<}\left(x_{0}, z\right)\right]
\end{aligned}
$$

where $\theta(x)$ is the unit step function $(\equiv 1,1 / 2,0$ for $x>0, \quad x=0$, and $x<0$, respectively) and the Wronskian

$$
W(z) \equiv\left|\begin{array}{cc}
G_{h}^{<}\left(x_{0}, z\right) & G_{h}^{>}\left(x_{0}, z\right) \\
\frac{\partial G_{h}^{<}\left(x_{0}, z\right)}{\partial x_{0}} & \frac{\partial G_{h}^{>}\left(x_{0}, z\right)}{\partial x_{0}}
\end{array}\right|
$$

is actually independent of $x_{0}$.

With the abbreviations

$$
\begin{aligned}
& x_{1} \equiv\left(-\frac{2 m}{\hbar^{2}} z\right)^{1 / 2}, \\
& x_{2} \equiv\left[\frac{2 m}{\hbar^{2}}\left(V_{0}-z\right)\right]^{1 / 2}, \\
& x_{3} \equiv \varkappa_{1}
\end{aligned}
$$

and defining intervals of the $x$-axis

$$
\begin{aligned}
& I_{1} \equiv(-\infty, 0), \\
& I_{2} \equiv[0, L], \\
& I_{3} \equiv(L,+\infty),
\end{aligned}
$$

equation (5) is written for $x \in I_{i}(i=1,2,3)$

$$
\frac{\partial^{2} G_{h}}{\partial x^{2}}-x_{i}^{2} G_{h}=0
$$

the general solution of which is given by

$$
G_{h}=A_{i} e^{\varkappa_{i} x}+B_{i} e^{-\varkappa_{i} x}
$$

with still undetermined constants $A_{i}, B_{i}$.

From the definitions

$$
\begin{aligned}
& \zeta_{1}(x) \equiv 1-\theta(x), \\
& \zeta_{2}(x) \equiv \theta(x)-\theta(x-L), \\
& \zeta_{3}(x) \equiv \theta(x-L)
\end{aligned}
$$

we have

$$
\zeta_{i}(x)= \begin{cases}1 & \text { for } x \in I_{i} \\ 0 & \text { else }\end{cases}
$$

and therefore for all $x$

$$
G_{h}=\sum_{i=1}^{3}\left(A_{i} e^{\varkappa_{i} x}+B_{i} e^{-\varkappa_{i} x}\right) \zeta_{i}(x) .
$$

The six constants $A_{i}, B_{i}(i=1,2,3)$ are interrelated by the well-known joint conditions for continuity and smoothness of $G_{h}$ at $x=0$ and $x=L$

$A_{1}+B_{1}=A_{2}+B_{2}$,

$\varkappa_{1}\left(A_{1}-B_{1}\right)=\varkappa_{2}\left(A_{2}-B_{2}\right)$,

$A_{2} e^{\varkappa_{2} L}+B_{2} e^{-\varkappa_{2} L}=A_{3} e^{\varkappa_{1} L}+B_{3} e^{-\varkappa_{1} L}$,

$\varkappa_{2}\left(A_{2} e^{\varkappa_{2} L}-B_{2} e^{-\varkappa_{2} L}\right)=\varkappa_{1}\left(A_{3} e^{\varkappa_{1} L}-B_{3} e^{-\varkappa_{1} L}\right)$

from which e.g. $A_{1}, B_{1}, A_{3}, B_{3}$ may be expressed as functions of $A_{2}, B_{2}$ (provided $\varkappa_{1} \neq 0$ ) and eliminated from (15). After some lengthy algebra we obtain

$$
G_{h}=A_{2} f(x, z)+B_{2} g(x, z)
$$

where

$$
\begin{aligned}
& f(x, z) \equiv \zeta_{1}(x)\left(\gamma_{+} e^{x_{1} x}+\gamma_{-} e^{-x_{1} x}\right)+\zeta_{2}(x) e^{\alpha_{2} x} \quad(18 \mathrm{a}) \\
& +\zeta_{3}(x)\left(\gamma_{+} e^{\left(x_{2}-x_{1}\right) L+x_{1} x}+\gamma_{-} e^{\left(\chi_{2}+\chi_{1}\right) L-\chi_{1} x}\right), \\
& g(x, z) \equiv \zeta_{1}(x)\left(\gamma_{-} e^{\alpha_{1} x}+\gamma_{+} e^{-x_{1} x}\right)+\zeta_{2}(x) e^{-x_{2} x} \\
& +\zeta_{3}(x)\left(\gamma_{-} e^{-\left(\varkappa_{2}+\varkappa_{1}\right) L+\chi_{1} x}+\gamma_{+} e^{-\left(\varkappa_{2}-\chi_{1}\right) L-\chi_{1} x}\right),
\end{aligned}
$$

and

$$
\gamma_{ \pm}(z) \equiv \frac{1}{2}\left[1 \pm \frac{x_{2}}{x_{1}}\right] .
$$

When the convention is adopted that the square root of a complex number $w=|w| e^{\mathrm{i} \varphi}(-\pi<\varphi \leqq \pi)$ is taken to be

$$
w^{1 / 2}=+\mid w^{1 / 2} \exp \{i \varphi / 2\}
$$

then we have for all $z$

$$
\begin{aligned}
& \operatorname{Re}\left(x_{1}\right) \geqq 0, \\
& \operatorname{Re}\left(x_{2}\right) \geqq 0,
\end{aligned}
$$

where the sign of equality holds only for real $z \geqq 0$ in (20a) and only for real $z \geqq V_{0}$ in (20b). Hence (except for real $z \geqq 0$ )

$$
\begin{aligned}
& \lim _{x \rightarrow-\infty}\left|e^{-x_{1} x}\right|=\lim _{x \rightarrow-\infty} e^{x \mid \operatorname{Re} x_{1}}=+\infty, \\
& \lim _{x \rightarrow+\infty}\left|e^{+x_{1} x}\right|=\lim _{x \rightarrow+\infty} e^{x \operatorname{Re} x_{1}}=+\infty .
\end{aligned}
$$

Thus the solutions $G_{h}^{>}, G_{h}^{<}$of (5) can be written

$$
G_{h}^{\gtrless}(x, z)=A_{2}^{\gtrless} f(x, z)+B_{2}^{\gtrless} g(x, z),
$$

where the boundary conditions $(6 \mathrm{a}, \mathrm{b})$ are satisfied if

$$
A_{2}>\gamma_{+} e^{\varkappa_{2} L}+B_{2}^{>} \gamma_{-} e^{-\varkappa_{2} L}=0
$$

and

$$
A_{2}^{<} \gamma_{-}+B_{2}^{<} \gamma_{+}=0 \text {. }
$$


When $B_{2}^{>}$and $B_{2}^{<}$are eliminated by the last two equations, $G_{h}^{>}$and $G_{h}^{<}$become proportional to $A_{2}^{>}$ and $A_{2}^{<}$, respectively. Then it is clear from (7), (8) that the product $A_{2}^{>} A_{2}^{<}$cancels in the expression for $G\left(x, x_{0}, z\right)$, so we can set $A_{2}^{>}=A_{2}^{<}=1$. With this convention we obtain from $(18 \mathrm{a}, \mathrm{b}),(19)$, (22), $(23 \mathrm{a}, \mathrm{b})$

$$
\begin{aligned}
& G_{h}^{>}(x, z)=\frac{2 e^{\varkappa_{2} L}}{r-z} g_{h}^{>}(x, z), \\
& g_{h}^{>}(x, z) \equiv \zeta_{1}(x)\left[\frac{V_{0}}{2} \sinh \left(\varkappa_{2} L\right) e^{\varkappa_{1} x}+t e^{-k_{1} x}\right] \\
& \quad+\zeta_{2}(x)\left[z \sinh \left(\varkappa_{2}(L-x)\right)+r \cosh \left(\varkappa_{2}(L-x)\right)\right] \\
& \quad+\zeta_{3}(x) r e^{\chi_{1}(L-x)}
\end{aligned}
$$

and

$$
\begin{aligned}
& G_{h}^{<}(x, z)=\frac{2}{r+z} g_{h}^{<}(x, z) \\
& g_{h}^{<}(x, z) \equiv \zeta_{1}(x) r e^{\varkappa_{1} x} \\
& \quad+\zeta_{2}(x)\left[z \sinh \left(\varkappa_{2} x\right)+r \cosh \left(\varkappa_{2} x\right)\right] \\
& \quad+\zeta_{3}(x)\left[\frac{V_{0}}{2} \sinh \left(\varkappa_{2} L\right) e^{-\varkappa_{1}(x-L)}+t e^{\varkappa_{1}(x-L)}\right]
\end{aligned}
$$

with

$$
\begin{aligned}
& r \equiv z\left(\varkappa_{2} / \varkappa_{1}\right), \\
& t \equiv\left(z-V_{0} / 2\right) \sinh \left(\varkappa_{2} L\right)+r \cosh \left(\varkappa_{2} L\right) .
\end{aligned}
$$

When expressions $(24 \mathrm{a}, \mathrm{b}),(25 \mathrm{a}, \mathrm{b})$ are inserted in eqn. (8), we find after a lot of algebra

$$
W(z)=8 \varkappa_{2} t e^{\varkappa_{2} L} / V_{0}
$$

and finally using (7)

$$
\begin{aligned}
& G\left(x, x_{0}, z\right)=-\frac{m}{\hbar^{2} z x_{2} t} \\
& \cdot\left[\theta\left(x-x_{0}\right) g_{h}^{>}(x, z) g_{h}^{<}\left(x_{0}, z\right)\right. \\
& \left.\quad+\theta\left(x_{0}-x\right) g_{h}^{<}(x, z) g_{h}^{>}\left(x_{0}, z\right)\right] .
\end{aligned}
$$

Let us discuss briefly the analytical structure of $G$. Along the positive real $z$-axis a branch cut of $G$ is found because $\varkappa_{1}$ appears in $G$. For $x>x_{0}$ we have $\zeta_{i}(x) \cdot \zeta_{j}\left(x_{0}\right)=0$ if $i<j$. A term-by-term inspection then shows that all nonvanishing products appearing in $g_{h}(x, z) \cdot g_{h}^{<}\left(x_{0}, z\right)$ tend to zero at least $\sim z^{1 / 2}$ for $z \rightarrow 0$. The same behaviour is found for $x<x_{0}$. Therefore $G$ has a singularity $\sim z^{-1 / 2}$ (not $\sim z$ ) for $z \rightarrow 0$.

When $z \rightarrow V_{0}, x_{2} \cdot t \sim\left(V_{0}-z\right)$. Since, however, the expression in square brackets is easily seen to be of the same order, $G$ remains finite for $z \rightarrow V_{0}$.
Apart from $z=V_{0}$, the equation $t=0$ turns out to have further solutions only if $z$ is real with $V_{0}<z<0$. Using the abbreviations $\vartheta \equiv L\left[2 m\left(z-V_{0}\right) / \hbar^{2}\right]^{1 / 2}$ and $v_{0} \equiv V_{0} L^{2} m / \hbar^{2}, t$ vanishes if $\vartheta$ satisfies

$$
\left(\vartheta^{2}+v_{0}\right) \sin \vartheta-\vartheta \sqrt{2\left|v_{0}\right|-\vartheta^{2}} \cos \vartheta=0
$$

which leads just to the transcendental eigenvalue equations for even and odd eigenfunctions which, e.g. in the notation of Flügge [18], read (with $2 a k \equiv \vartheta$ and $C^{2} \equiv-v_{0} / 2$ )

$$
a k \tan (a k)=\sqrt{C^{2}-a^{2} k^{2}}
$$

and

$$
\sqrt{C^{2}-a^{2} k^{2}} \tan (a k)=-a k .
$$

Thus the poles of $G$ determine the exact bound state energies as is required from the spectral representation (3).

Finally we show how the exact Green's functions for the special cases of the free particle $\left(V_{0} \rightarrow 0\right)$ and of the particle in a box with infinitely high walls are recovered from expression (28).

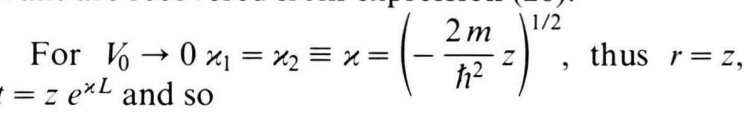

$$
\begin{aligned}
g^{>}(x, z) & =z e^{x(L-x)}\left(\zeta_{1}(x)+\zeta_{2}(x)+\zeta_{3}(x)\right) \\
& =z e^{x(L-x)}
\end{aligned}
$$

and analogously

$$
g^{<}(x, z)=z e^{x x} .
$$

Therefore we get from (28) the free particle Green's function

$$
\begin{aligned}
\lim _{V_{0} \rightarrow 0} G\left(x, x_{0}, z\right)= & -\frac{m}{\hbar^{2} \varkappa}\left[\theta\left(x-x_{0}\right) e^{\varkappa\left(x_{0}-x\right)}\right. \\
& \left.+\theta\left(x_{0}-x\right) e^{-\varkappa\left(x_{0}-x\right)}\right] \\
= & -\frac{m}{\hbar^{2} \varkappa} e^{-\varkappa\left|x-x_{0}\right|} .
\end{aligned}
$$

The Green's function for the box with infinitely high walls is obtained from the Green's function $G_{1}$ for the potential

$$
V_{1}(x) \equiv V(x)-V_{0}=V(x)+\left|V_{0}\right|
$$

in the limit $\left|V_{0}\right| \rightarrow \infty\left(V_{0}<0\right)$. Since, however,

$$
\begin{aligned}
(z & \left.-\hat{H}_{1 x}\right) G_{1}\left(x, x_{0}, z\right) \\
& =\left[z-\left(-\frac{\hbar^{2}}{2 m} \frac{\partial^{2}}{\partial x^{2}}+V(x)-V_{0}\right)\right] G_{1}\left(x, x_{0}, z\right) \\
& =\left[\left(z+V_{0}\right)-\hat{H}_{x}\right] G_{1}\left(x, x_{0}, z\right)=\delta\left(x-x_{0}\right)
\end{aligned}
$$


it follows that

$$
G_{1}\left(x, x_{0}, z\right)=G\left(x, x_{0}, z+V_{0}\right) .
$$

Therefore we have to replace $z$ by $z+V_{0}$ in expression (28) and then let $V_{0} \rightarrow-\infty$. When $\varkappa_{1}=$ $\left[-\alpha\left(z+V_{0}\right)\right]^{1 / 2}, \varkappa_{2}=(-\alpha z)^{1 / 2}\left(\alpha \equiv 2 m / \hbar^{2}\right)$ are inserted in (25a, b), (26a, b), and (28), we obtain for $x>x_{0}$ in the limit $V_{0} \rightarrow-\infty$

$$
\begin{aligned}
g_{h}(x) g_{h}^{<}\left(x_{0}\right)= & \left(z+V_{0}\right)^{2} \zeta_{2}(x) \zeta_{2}\left(x_{0}\right) \\
& \cdot \sinh \left[\varkappa_{2}(L-x)\right] \sinh \left(\varkappa_{2} x_{0}\right) .
\end{aligned}
$$

All the other terms tend to zero exponentially. The diverging term $\left(z+V_{0}\right)^{2}$ cancels a corresponding term in the denominator of $G\left(x, x_{0}, z+V_{0}\right)$. If we write $(-\alpha z)^{1 / 2}=i(\alpha z)^{1 / 2}$ noting $0<\operatorname{Re}(-\alpha z)^{1 / 2}$ $=-\operatorname{Im}(\alpha z)^{1 / 2}$ we finally obtain

$$
\begin{aligned}
\lim _{V_{0} \rightarrow-\infty} & G\left(x, x_{0}, z+V_{0}\right)=\frac{\alpha^{1 / 2}}{z^{1 / 2} \sin \left(\alpha^{1 / 2} L\right)} \\
\times & {\left[\theta\left(x-x_{0}\right) \sin \left(\alpha^{1 / 2}(x-L)\right) \sin \left(\alpha^{1 / 2} x_{0}\right)\right.} \\
+ & \left.\theta\left(x_{0}-x\right) \sin \left(\alpha^{1 / 2}\left(x_{0}-L\right)\right) \sin \left(\alpha^{1 / 2} x\right)\right]
\end{aligned}
$$

where now $0 \leqq x, x_{0} \leqq L$.

[1] E. N. Economou, Green's Functions in Quantum Physics, Springer Series in Solid-State Sciences 7, Springer-Verlag, Berlin, New York 1979.

[2] N. H. March and J. C. Stoddart, Rep. Progr. Phys. XXXI, 533 (1968).

[3] P. Roman, Advanced Quantum Theory, AddisonWesley Publ. Comp. Inc., Reading, Mass. 1965.

[4] R. Courant and D. Hilbert, Methods of Mathematical Physics, Vol. I, Interscience Publ. Inc., New York 1953.

[5] P. M. Morse and H. Feshbach, Methods of Theoretical Physics, 2 Vols., McGraw-Hill, New York 1953.

[6] V. L. Bachrach, S. I. Vetchinkin, and S. V. Khristenko, Teor. Mat. Fiz. 12, 776 (1972).

[7] S. I. Vetchinkin and V. L. Bachrach, Int. J. Quantum Chem. VI, 143 (1972).

[8] G. Berendt and E. Weimar, Lett. Nuovo Cim. 5, 613 (1972).

[9] J. Bellandi Fo and E. S. Caetano Neto, J. Phys. A9, 683 (1976).
This limit agrees with the Green's function for the box with infinitely high walls as given e.g. by Morse-Feshbach [5] if we identify $\lambda=\alpha z, G_{\lambda}\left(x \mid x_{0}\right)$ $=-\frac{4 \pi}{\alpha} \lim _{V_{0} \rightarrow-\infty} G\left(x, x_{0}, z+V_{0}\right), \quad b=L, a=0$ where the lihs notations are used by these authors.

\section{Acknowledgement}

Financial support by the "Fonds der Chemischen Industrie" is gratefully acknowledged.

\section{Note added in proof:}

Only recently the author became aware of a paper of J. L. Beeby, Proc. Camb. Phil. Soc. 59, 609 (1963), where the propagator for a square well $\left(V_{0}<0\right)$ has been calculated by a similar method for the special case of $\operatorname{Im}(z) \rightarrow \pm 0$.

[10] E. H. Wichmann and C.-H. Woo, J. Math. Phys. 2, $178(1961)$

[11] J. Schwinger, J. Math. Phys. 5, 1606 (1964).

[12] L. Hostler, J. Math. Phys. 5, 591 (1964); 5, 1235 (1964).

[13] V. Skobov, Sov. Phys. - JETP 10, 1039 (1960).

[14] G. Gountaroulis, Phys. Lett. 40 A, 132 (1972).

[15] S. M. Blinder, Configuration-space Green's functions, in International Review of Science 1, 21 (London 1975).

[16] R. P. Feynman and A. R. Hibbs, Quantum Mechanics and Path Integrals, McGraw-Hill Book Comp., New York 1965.

[17] W. Janke and H. Kleinert, Lett. Nuovo Cim. 25, 297 (1979).

[18] S. Flügge, Practical Quantum Mechanics, SpringerVerlag, New York 1974, p. 50, Eqs. (25.7e), (25.7o). 